\title{
Ricci Collineations
}

\author{
for \\ type B warped space-times
}

\author{
J.Carot* \\ Departament de Física \\ Universitat de les Illes Balears \\ E-07071 Palma de Mallorca \\ Spain \\ L. A. Núñez ${ }^{\dagger}$ and U. Percoco ${ }^{\ddagger}$ \\ Centro de Astrofísica Teórica \\ Departamento de Física, Facultad de Ciencias, \\ Universidad de los Andes, Mérida 5101, Venezuela.
}

July 31, 2021

\begin{abstract}
We present the general structure of proper Ricci Collineations (RC) for type B warped space-times. Within this framework, we give a detailed description of the most general proper $\mathrm{RC}$ for spherically symmetric metrics. As examples, static spherically symmetric and Friedmann-Robertson-Walker space-times are considered.
\end{abstract}

\footnotetext{
*Email: dfsjcg0@ps.uib.es

†Email:nunez@ciens.ula.ve

†Email: upercoco@ciens.ula.ve
} 


\section{Introduction}

The purpose of this paper is to study Ricci Collineations (RC) for a certain class of space-times, namely type B warped space-times and in particular spherically symmetric space-times. Collineations are symmetry properties of space times. Katzin et al. [1] define them as those vector fields, $X$, such that leave the various relevant geometric quantities in General Relativity invariant under Lie dragging in their direction. The best known examples of collineations are the Killing vectors (Motions), i.e. vectors that satisfy:

$$
£_{X} g_{a b}=0
$$

Other interesting symmetries are defined in analogously and the more frecuent cases of study have been:

Conformal Motions:

$$
£_{X} g_{a b}=2 \sigma g_{a b}
$$

Affine Collineations:

$$
£_{X} \Gamma_{a b}^{c}=0
$$

Curvature Collineations:

$$
£_{X} R_{a b c d}=0
$$

Ricci Collineations:

$$
£_{X} R_{a b}=0
$$

Contracted Ricci Collineations:

$$
g^{a b} £_{X} R_{a b}=0
$$

Here $£_{X}$ stands for the Lie derivative operator and the indices $a, b, \ldots$ run from 1 to 4 .

The well established connection between Killing Vectors and constants of the motion has encouraged the search for general relations between collineations and conservation laws. Collineations, other than Motions, can be considered as non-noetherian symmetries and can also be associated to constants of the motion. Affine Collineations have been shown to be related to conserved quantities [ [Q], and this property has been used to integrate geodesics of the Robertson-Walker metric [3]. As far as we know, the first Curvature Collineation was found by Aichelburg [4 for pp-wave metrics, and their relationships to first integrals of the geodesic equations extensively studied in 
Ref. [1]. Particular types of Ricci and Contracted Ricci Collineations, for the Robertson-Walker metric have also been found and shown to be related to the particle number conservation [5]. Also, considerable attention is being paid to the related problem of symmetry inheritance in General Relativity [6]. Collineations have been studied in connection with fluid spacetimes [5], [7], [8], [9] and some specific examples have been given for the $C$-metric [10], Robertson-Walker Spacetimes [11], and Gödel-type manifolds [12].

It is clear from the above definitions that Motions are particular cases of Affine Collineations, Affine Collineations are particular cases of Curvature Collineations, and so on. It is therefore possible to construct an "inclusion diagram" connecting these symmetries. One such diagram, that includes these and other related symmetries, is presented in Ref. [1]. A collineation of a given type is said to be proper if it does not belong to any of the subtypes. Clearly, in solving any collination equation, with the obvious exception of the Killing equation, solutions representing improper collineations can be found. Therefore, in order to relate a symmetry to a particular conservation law, and its corresponding constant of the motion, the "properness" of that collineation must be assured. Some computer algebra tools have been developed to check the properness of Ricci and other collineation vectors are under development [13] [14].

We assume that RCs are smooth vector fields. Although this is not necessarily so, by restricting ourselves to this case, we ensure that they form a Lie algebra with the usual bracket operation. Such an algebra naturally contains that of Special Conformal Killing Vectors (SCKV) (see reference [6]) which in turn contains that of Homothetic Vector Fields (HVF) and therefore the isometry algebra of all Killing Vector Fields (KV).

Regarding the Ricci tensor, we shall consider that it is non-degenerate (i.e.: rank 4) and this in turn ensures that the Lie algebra of RC is finite dimensional, its maximal dimension being 10 (9 being forbidden by Fubini 's theorem). For further information on issues concerning dimensionality and degenerate Ricci tensor see, for instance, references [15. and 17.

The paper is organized as follows: in section 2 we describe the basic features of the RC in type $\mathrm{B}$ warped space-times, then, in section 3, we consider spherically symmetric space-times as a particular case of these, studying two distinct cases, namely: static solutions and Friedmann-Robertson-Walker space-times. 


\section{Type B warped space-times}

Suppose that $\left(M_{1}, h_{1}\right)$ and $\left(M_{2}, h_{2}\right)$ are a pair of pseudo-Riemannian manifolds, and $\Phi$ is a real valued function on $M_{1}$ ('warping function'), one can then build a Lorentz manifold, $(M, g)$ by setting $M=M_{1} \times M_{2}$ and $g=\pi_{1}^{*} h_{1} \otimes \Phi^{2} \pi_{2}^{*} h_{2}$, where the functions $\pi_{1}$ and $\pi_{2}$ are the canonical projections onto the factors of the product. $(M, g)$ is then called a 'warped product manifold'. If $\operatorname{dim} M=4$, we say that $(M, g)$ is a 'warped space-time' and one can classify them according to the respective dimensions of the factor (sub-)manifolds $M_{1}$ and $M_{2}$. We shall refer the reader to [18] and references cited therein for a general discussion, restricting ourselves hereafter to the case $\operatorname{dim} M_{1}=\operatorname{dim} M_{2}=2$, namely; warped space-times of the class $B$. Although all our considerations will be local, see [19 for some remarks on globally warped space-times. It can be shown that for type $B$ warped spacetimes, a coordinate chart exists (adapted to the manifold product structure), such that the metric takes the form

$$
d s^{2}=h_{A B}\left(x^{D}\right) \mathrm{d} x^{A} \mathrm{~d} x^{B}+\Phi^{2}\left(x^{D}\right) h_{\alpha \beta}\left(x^{\gamma}\right) \mathrm{d} x^{\alpha} \mathrm{d} x^{\beta}
$$

where the indices $A, B, \ldots$ run from 1 to 2 and $\alpha, \beta, \ldots$ from 3 to 4 . The functions $h_{A B}$ and $h_{\alpha \beta}$ are the component forms of $\pi_{1}^{*} h_{1}$ and $\pi_{2}^{*} h_{2}$ in the local charts $\left\{x^{A}\right\}$ and $\left\{x^{\alpha}\right\}$, which are in turn adapted to $M_{1}$ and $M_{2}$ respectively. The Ricci tensor of such a space-time takes then the following component form in the above chart:

$$
\begin{gathered}
R_{A B}=\frac{1}{2} R_{1} h_{A B}-\frac{2}{\Phi} \Phi_{A ; B}, \\
R_{A \alpha}=0 \\
R_{\alpha \beta}=\frac{1}{2}\left(R_{2}-\left(\Phi^{2}\right)_{; A}^{A}\right) h_{\alpha \beta} \equiv F h_{\alpha \beta} ;
\end{gathered}
$$

where $F=\frac{1}{2}\left(R_{2}-\left(\Phi^{2}\right)_{; A}^{A}\right)$ and $R_{1}$ and $R_{2}$ are the Ricci scalars associated to the 2-metrics $h_{1}$ and $h_{2}$. The semi-colon indicates, as usual, the covariant derivative with respect to the space-time metric.

Let now $X$ be a $\mathrm{RC}$ on $M$, and define its vertical and horizontal components, $X_{1}$ and $X_{2}$, as follows (see [18]):

$$
X_{1}^{a} \equiv g^{a b}\left(\pi_{1}^{*} h_{1}\right)_{b d} X^{d} \quad X_{2}^{a} \equiv X^{a}-X_{1}^{a}
$$


In the above adapted chart, one readily sees that $X_{1}^{A}=X^{A}, X_{1}^{\alpha}=0$, and $X_{2}^{A}=0, X_{2}^{\alpha}=X^{\alpha}$.

On account of (8), (9) , (10) and (11), equation (5) is now equivalent to:

$$
\begin{gathered}
R_{A B, D} X_{1}^{D}+R_{A D} X_{1, B}^{D}+R_{D B} X_{1, A}^{D}=0, \\
R_{A D} X_{1, \alpha}^{D}+F h_{\alpha \beta} X_{2, A}^{\beta}=0 \\
£_{X_{2}} h_{\alpha \beta}=2 \Psi h_{\alpha \beta}
\end{gathered}
$$

where

$$
\Psi=-\frac{1}{2} \frac{F_{, D} X_{1}^{D}+F_{, \gamma} X_{2}^{\gamma}}{F}
$$

Take now $p_{1} \in M_{1}$ and consider the manifold $\tilde{M}_{2} \equiv\left\{p_{1}\right\} \times M_{2} \cong M_{2}$ (see [18]), equation (14) is then a statement that $X_{2}$ is a Conformal Killing Vector (CKV) of $\left(\tilde{M}_{2}, h_{2}\right)$, and therefore it can be re-written as

$$
X_{2 \alpha / \beta}+X_{2 \beta / \alpha}=2 \Psi h_{\alpha \beta}
$$

where a stroke denotes the covariant derivative associated with the metric $h_{2}$.

Furthermore, it is possible to write [16,

$$
£_{X_{2}} R_{2 \alpha \beta}=-2 \Psi_{\alpha / \beta}-\left(h^{\mu \nu} \Psi_{\mu / \nu}\right) h_{\alpha \beta}
$$

where

$$
R_{2 \alpha \beta}=\frac{R_{2}}{2} h_{\alpha \beta}
$$

is the Ricci tensor of the metric $h_{2}$. In addition, the Conformal Bivector associated to $X_{2}$, i.e.

$$
F_{\alpha \beta} \equiv X_{2 \alpha / \beta}-X_{2 \beta / \alpha}
$$

satisfies

$$
F_{\alpha \beta / \gamma}=\frac{R_{2}}{2}\left(h_{\alpha \gamma} X_{2 \beta}-X_{2 \alpha} h_{\beta \gamma}\right)-\Psi_{\alpha} h_{\beta \gamma}+\Psi_{\beta} h_{\alpha \gamma}
$$

Now, from (17) one obtains

$$
\Psi_{\alpha / \beta}=\lambda h_{\alpha \beta} \quad \text { and } \quad \lambda \equiv-\frac{1}{8}\left(£_{X_{2}} R_{2}+2 \Psi R_{2}\right)
$$


and from the Bianchi identities (on $\left(\tilde{M}_{2}, h_{2}\right)$ ) for $\Psi$, it readily follows:

$$
\lambda_{, \gamma}=-\frac{R_{2}}{2} \Psi_{, \gamma}
$$

Furthermore, taking a further covariant derivative in the above expression, skewsymmetrising, and equating to zero, one has

$$
-\left(\frac{R_{2}}{2}\right)_{, \alpha}=\sigma \Psi_{, \alpha}
$$

for some function $\sigma$.

To proceed with our study, it is useful to consider now the following decomposition of $\tilde{M}_{2} ; \tilde{M}_{2}=H \cup K \cup C$, where $H$ is that open submanifold of $\tilde{M}_{2}$ on which $\Psi_{\alpha / \beta} \Leftarrow 0$ (hence $\lambda \nRightarrow 0$ and $\Psi^{\alpha} \Psi_{\alpha} \Leftarrow 0$ on $H$ ), $K$ is the interior of that set of points for which $\Psi_{\alpha / \beta}=0$, and $C$ is a set with no interior defined by the decomposition itself.

We shall first study what happens in $K$. Since $\Psi_{\alpha / \beta}=0$ there, it follows that $\Psi_{, \alpha}$ is either zero on $K$ (in which case $X_{2}$ is homothetic), or else it is a (gradient) Killing vector (and then $X_{2}$ is an SCKV), the Bianchi identities then implying $R_{2}=0$, i.e.; $\left.h_{2}\right|_{K}$ is flat. In the latter case ( $h_{2}$ flat), one can always choose coordinates on $K$, say $\{x, y\}$, such that $\left.\Psi\right|_{K}=A x(A=$ constant), and integrating out the conformal equations (14) for $X_{2}$ on $K$ it follows

$$
X_{2}=\left(\frac{1}{2} A\left(x^{2}-y^{2}\right)-D y+L\right) \partial_{x}+(A x y+D x+E) \partial_{y}
$$

where $A, D, E$ and $L$ are constants on $K$ which will, in general, depend on the chosen $p_{1} \in M_{1}$, and therefore, when considering $X_{2}$ on $M$, one will have that all of them are functions of the coordinates set up in $M_{1}$, thus $A=A\left(x^{B}\right), D=D\left(x^{B}\right), \cdots$ to be determined, along with the vertical component $X_{1}$ of $X$, from (12) and (13). In fact, it is easy to see from (13) that $A$ and $D$ must be constants, say $A=A_{0}$ and $D=D_{0}$, and from the expression (15) of $\Psi$ with $R_{2}=0$, together with $\Psi=A_{0} x$, it follows that $E$ must also be constant (which can be set equal to zero without loss of generality), then from ([12) $X_{1}^{A}=P^{A}\left(x^{B}\right) x+Q^{A}\left(x^{B}\right)$, and therefore one has, on $M \cap K$ and if $R_{2}=0$ :

$$
X=\left(P^{A} x+Q^{A}\right) \partial_{A}+\left(\frac{A_{0}}{2}\left(x^{2}-y^{2}\right)-D_{0} y+L\right) \partial_{x}+\left(A_{0} x y+D_{0} x\right) \partial_{y}
$$




$$
\Psi=A_{0} x
$$

$P, Q$ and $L$ being functions of the coordinates $\left\{x^{B}\right\}$ on $M_{1}$ to be determined from $(12)$ and (13).

If $\left.\Psi_{, \alpha}\right|_{K}=0, X_{2}$ is an HVF, and therefore $([16]) £_{X_{2}} R_{2}=-2 \Psi R_{2}$ if $R_{2} \neq$ constant or $£_{X_{2}} R_{2}=0$ if $R_{2}=$ constant, hence (15) implies

$$
\Psi=-\frac{1}{2} \frac{\left(\Phi^{2}\right)_{; A D}^{A} X_{1}^{D}}{\left(\Phi^{2}\right)_{; A}^{A}}
$$

thus, given a basis of the homothetic algebra of $\left(M_{2}, h_{2}\right)$, say $\left\{\zeta_{I}\right\}$ with $I \leq 4$, one will have $X_{2}=C^{I} \zeta_{I}$ on $\left(\tilde{M}_{2}, h_{2}\right)$, the $C^{I}$ being constants which will in general depend on the chosen $p_{1} \in M_{1}$, and again, when considering $X_{2}$ on $M$, they will become functions of the coordinates in $M_{1}$, to be determined as before from (12) and (13). It is worth noticing that, whenever a proper HVF exists in $\left(\tilde{M}_{2}, h_{2}\right)$, say $\zeta_{1}$ then (14) implies that $C^{1}=\Psi$. It will be shown later on that, in all cases but one, the functions $C^{I}$ must in fact be constants (and (13) then implies that $X_{1}$ is just a vector field on $M_{1}$ ). Thus, we conclude that whenever $\Psi_{, \alpha}=0$, one has

$$
X=X_{1}^{A}\left(x^{B}, x^{\gamma}\right) \partial_{A}+C^{I}\left(x^{B}\right) \zeta_{I}
$$

where $C^{I}$ and $X_{1}^{A}$ are functions of their arguments, to be determined from (12) and (13), and $\left\{\zeta_{I}\right\}$ with $I \leq 4$ form a basis of the homothetic algebra of $\left(M_{2}, h_{2}\right)$.

Let us next study what happens on $H$. Notice that (21) can be rewritten as $£_{Y} h_{\alpha \beta}=2 \lambda h_{\alpha \beta}$ with $Y_{\alpha}=\Psi_{, \alpha}$; thus, $Y$ is also a CKV of $\left(\tilde{M}_{2}, h_{2}\right)$ with conformal factor $\lambda$, and one therefore has [16]:

$$
£_{Y} R_{2 \alpha \beta}=-2 \lambda_{\alpha / \beta}-\left(h^{\mu \nu} \lambda_{\mu / \nu}\right) h_{\alpha \beta}
$$

which, on account of (18) and (21), can be rewritten as

$$
\lambda_{\alpha / \beta}=-\frac{1}{8}\left(R_{2, \alpha} \Psi^{\alpha}+2 \lambda R_{2}\right) h_{\alpha \beta} \equiv \Sigma h_{\alpha \beta}
$$

that is: $Z$ such that $Z_{\alpha} \equiv \lambda_{, \alpha}$ is a (gradient) CKV, colinear with another $\mathrm{CKV}$, namely $Y$; it is then immediate to show, taking into account (21), (22), (23) and (30), that $R_{2}$ must be constant $(\sigma=0$ in (23)), (21) then reading

$$
\Psi_{\alpha / \beta}=-\frac{R_{2}}{4} \Psi h_{\alpha / \beta}
$$

The Bianchi identities specialized to $\Psi_{, \alpha}$ then imply one of the following: 
1. $R_{2}=0$ and $\Psi_{, \alpha} \Leftarrow 0$, one then has the expression (24) for $X_{2}$, etc.

2. $R_{2}=$ constant $(\bumpeq 0)$ and $\Psi_{, \alpha}=0, X_{2}$ is then an HVF of $\left(\tilde{M}_{2}, h_{2}\right)$, but since $R_{2}$ is constant and non-zero, it must be a $\mathrm{KV}$, i.e.; $\Psi=0$.

3. $R_{2}=0$ and $\Psi_{, \alpha}=0, X_{2}$ is an $\operatorname{HVF}$ of $\left(\tilde{M}_{2}, h_{2}\right)$, possibly non-Killing.

Notice that whenever $\Psi_{, \alpha}=0$, one gets the same results as when studying this case in $K \subset \tilde{M}_{2}$, i.e.; equations (27) and (28) hold.

We can roughly summarize the results so far obtained as follows:

The horizontal component $X_{2}$ of a RC $X$ is either an HVF of $\left(\tilde{M}_{2}, h_{2}\right)$ (i.e.; $\left.\Psi_{, \alpha}=0\right)$ and $X$ is then given by (28), or else it is a proper SCKV of $\left(\tilde{M}_{2}, h_{2}\right)$ (that is; $\Psi_{, \alpha} \models 0, \Psi_{\alpha / \beta}=0$ ), this being possible only when $R_{2}=0$ (i.e.; $\left(\tilde{M}_{2}, h_{2}\right)$ flat), and in that case $X$ takes the form given by (24). In both cases, the functions appearing in (28) and (24) must satisfy (12) and (13).

We shall next focus our attention on the case $X_{2}$ homothetic, studying the various cases that may arise in connection with the different structures and dimensions of the homothetic algebra of $\left(\tilde{M}_{2}, h_{2}\right)$.

To this end, let $\mathcal{H}_{r}$ be the homothetic algebra of $\left(\tilde{M}_{2}, h_{2}\right), r$ being its dimension. Since $\operatorname{dim} M_{2}=2$ it follows that $r$ can only be $0,1,2,3$ or 4. We shall deal separately with all these cases assuming, for the sake of simplicity, that $h_{2}$ is Riemannian (similar conclusions hold if $h_{2}$ is Lorentz).

1. $r=0$. In this case no HVFs exist (including $\mathrm{KVs}$ ), and therefore $\Psi=C^{I}=0$, i.e.; $X_{2}=0$ and $X=X_{1}$ with $X_{1, \alpha}^{D}=0$ as a consequence of (13), that is: $X$ is a vector field on $M_{1}$ which must satisfy (12) and (27) with $\Psi=0$.

2. $r=1$. There are now two cases to be distinguished, depending on whether a proper HVF exists or not.

(a) A proper HVF $\zeta$, exists in $\left(\tilde{M}_{2}, h_{2}\right)$. It is easy to see that one can then always choose coordinates, say $x$ and $y$, such that the line element $d \sigma^{2}$ associated with $h_{2}$, and the $\operatorname{HVF} \zeta$ read in these coordinates

$$
d \sigma^{2}=e^{2 y}\left(d x^{2}+h^{2}(x) d y^{2}\right) \text { and } \zeta=\partial_{y}
$$


the associated Ricci scalar is $R_{2}=-2 e^{-2 y} h^{-1} h^{\prime \prime}$ (a prime denoting derivative with respect to $x$ ), and (13) then implies:

$$
R_{A D} X_{1, x}^{D}=0 \quad \text { and } \quad R_{A D} X_{1, y}^{D}=-F \Psi_{, A} e^{2 y} h^{2}(x)
$$

which cannot be fulfilled unless $\left(F h^{2}(x)\right)_{, x}=0$, i.e.; $h(x)=$ constant, in which case $R_{2}=0$ and therefore $r=4$. Thus, $\Psi_{, A}=0$ $(\Psi=$ constant $\Leftarrow 0), X_{1, \alpha}^{D}=0$ and then $X=X_{1}^{A}\left(x^{D}\right) \partial_{A}+\Psi \zeta$ with $X_{1}$ satisfying (12) and (27) with $\Psi=$ constant $(\vDash 0)$.

(b) No proper HVF exists in $\left(\tilde{M}_{2}, h_{2}\right)$, just a $\mathrm{KV}$, say $\xi$. It then follows that $\Psi=0$ necessarily, and again coordinates may be chosen such that

$$
d \sigma^{2}=d x^{2}+h^{2}(x) d y^{2} \text { and } \xi=\partial_{y}
$$

the Ricci scalar is then $R_{2}=-2 h^{-1} h^{\prime \prime}$, and (13) implies, as in the previous case, $\left(F h^{2}(x)\right)_{, x}=0$, which in turn can be seen to imply

$$
\begin{aligned}
& \left(\Phi^{2}\right)_{; A}^{A}=2 a \quad, \quad(a=\text { constant }) \\
& h^{\prime \prime}+a h^{2}=b \quad, \quad(b=\text { constant })
\end{aligned}
$$

Performing now the coordinate change $h(x) \equiv z$, the above line element reads

$$
d \sigma^{2}=\frac{d z^{2}}{2 C+z^{2}+2 \log z}+z^{2} d y^{2}
$$

Hence (13) implies $X_{1}^{A}=P^{A}\left(x^{D}\right) y+Q^{A}\left(x^{D}\right)$ and then

$$
X=\left(P^{A}\left(x^{D}\right) y+Q^{A}\left(x^{D}\right)\right) \partial_{A}+C\left(x^{D}\right) \xi
$$

where $P^{A}\left(x^{D}\right)$ and $Q^{A}\left(x^{D}\right)$ must both satisfy (12) separately, and $C\left(x^{D}\right)$ must be such that $R_{A D} P^{D}=-b C_{, A}$.

3. $r=2, \mathcal{H}$ must then contain at least one proper HVF, since otherwise ( $\mathcal{H}$ spanned by two KVs) a third KV would necessarily exist, hence dim $\mathcal{H}=3$. Suppose then that a proper HVF, $\zeta$, exists; the other vector in the basis of $\mathcal{H}$ can always be chosen to be a $\mathrm{KV}$, say $\xi$, and there are two possible, non-isomorphic, Lie algebra structures for $\mathcal{H}$, namely 
$[\xi, \zeta]=0$ (abelian), and $[\xi, \zeta]=\xi$ (non abelian). In the abelian case, coordinates may be chosen such that the line element, $\zeta$ and $\xi$ read respectively

$$
d \sigma^{2}=d x^{2}+x^{2} d y^{2}, \zeta=x \partial_{x}, \xi=\partial_{y}
$$

but it then follows that $R_{2}=0$ and therefore two other KVs exist, $r$ thus being 4 , therefore this case cannot arise.

In the non-abelian case, and again by means of a suitable choice of coordinates, one has:

$$
d \sigma^{2}=d x^{2}+x^{2 \frac{n-1}{n}} d y^{2}, \zeta=n x \partial_{x}+y \partial_{y}(n \nprec 1), \xi=\partial_{y}
$$

but then (13) implies, as in previous cases, that $\left(F x^{2 \frac{n-1}{n}}\right)_{, x}=0$, which can not be satisfied, therefore $\Psi_{, A}=C_{A_{A}}=0$ (i.e.; $\Psi$ and $C$ constants) and then $X_{1, \alpha}^{D}=0$, and again $X=X_{1}^{A}\left(x^{D}\right) \partial_{A}+\Psi \zeta$ with $X_{1}$ satisfying (12) and (27) with $\Psi=$ constant $(\not 00)$.

4. $r=3$ If a proper HVF exists in $\left(\tilde{M}_{2}, h_{2}\right)$, the associated Killing subalgebra is then of dimension 2, and therefore a third KV exists, hence dim $\mathcal{H}=4$ and therefore this case is not possible. If, on the other hand, no proper HVFs exist, $\left(\tilde{M}_{2}, h_{2}\right)$ is of constant curvature and $\Psi=0$ necessarily. Let $\left\{\xi_{J}\right\}, J=1,2,3$ be three $\mathrm{KVs}$ spanning $\mathcal{H}$, from (13) it follows $R_{A D} X_{1, \alpha}^{D}=-F C_{, A}^{J} \xi_{J \alpha}$, differentiating with respect to $x^{\beta}$, skewsymmetrising and equating to zero, one has

$$
C_{, A}^{J} \xi_{J[\alpha, \beta]}=0
$$

that is; either $C_{, A}^{J}=0$ or else $\left(\tilde{M}_{2}, h_{2}\right)$ contains a gradient $\mathrm{KV}$. From [20], it is easy to see that the later is only possible if $R_{2}=0$, but in that case a proper HVF is always admitted (namely $\zeta=x \partial_{x}$ in the coordinates used in 20]), and therefore $\operatorname{dim} \mathcal{H}=4$.

5. $r=4$. In this case $\left(\tilde{M}_{2}, h_{2}\right)$ is flat, the line element and KVs being those given in 20] and the proper $\operatorname{HVF} \zeta=x \partial_{x}$. Proceeding as before, one can readily see from (13) that $X_{1}^{A}=M^{A}\left(x^{D}\right) x^{2}+\left(P_{1}^{A}\left(x^{D}\right) \cos y+\right.$ $\left.P_{3}^{A}\left(x^{D}\right) \sin y\right) x+Q^{A}\left(x^{D}\right)$, but since $\Psi \neq 0$ and $\Psi_{, \alpha}=0$, it follows that $P_{1}^{A}=P_{3}^{A}=M^{A}=0$, which in turn imply $\Psi_{, A}=C_{, A}^{1}=C_{{ }_{A}}^{2}=C_{{ }_{A}}^{3}=0$, 
hence $X_{1, \alpha}^{A}=0$, that is: $X_{1}$ is a vector field on $M_{1}$ that has to satisfy (12) and (27) with $\Psi=$ constant $(\models 0)$, and $X=X_{1}^{A}\left(x^{D}\right) \partial_{A}+\Psi \zeta+$ $C^{J} \xi_{J}$.

Our purpose in the next sections is to apply the results so far obtained to the case of spherically symmetric space-times which are also static, as well as to Friedmann-Robertson-Walker (FRW) models.

\section{Spherically symmetric space-times}

We next specify the above results to the case of a general spherically symmetric spacetime whose metric, in the local chart $\left\{x^{0,1,2,3}=t, r, \vartheta, \phi\right\}$ takes the form [20]

$$
d s^{2}=-\mathbf{e}^{2 \nu(t, r)} \mathrm{d} t^{2}+\mathbf{e}^{2 \lambda(t, r)} \mathrm{d} r^{2}+r^{2}\left(\mathrm{~d} \vartheta^{2}+\sin ^{2} \phi \mathrm{d} \phi^{2}\right)
$$

Comparing the metric (77) with the above (42), we have $\left\{x^{A}=t, r ; x^{\alpha}=\right.$ $\vartheta, \phi\} ; \Phi=r$;

$$
h_{A B}(t, r) \mathrm{d} x^{A} \mathrm{~d} x^{B}=-\mathbf{e}^{2 \nu(t, r)} \mathrm{d} t^{2}+\mathbf{e}^{2 \lambda(t, r)} \mathrm{d} r^{2}
$$

and

$$
h_{\alpha \beta} \mathrm{d} x^{\alpha} \mathrm{d} x^{\beta}=\mathrm{d} \vartheta^{2}+\sin ^{2} \phi \mathrm{d} \phi^{2}
$$

Thus, the Ricci tensor can be written as

$$
\begin{gathered}
R_{t t}=-\frac{1}{2} R_{1} \mathbf{e}^{2 \nu(t, r)}+\frac{2 \nu^{\prime}}{r} \mathbf{e}^{2(\nu(t, r)-\lambda(t, r))} \\
R_{t r}=\frac{2}{r} \dot{\lambda} \\
R_{r r}=\frac{1}{2} R_{1} \mathbf{e}^{2 \lambda}+\frac{2 \lambda^{\prime}}{r}
\end{gathered}
$$

and

$$
R_{\alpha \beta}=\left\{1-\mathbf{e}^{-2 \lambda}\left[1+r\left(\nu^{\prime}-\lambda^{\prime}\right)\right]\right\} h_{\alpha \beta}
$$

where a dash and a dot indicate, as usual, partial derivatives with respect to $r$ and $t$ respectively. As above, $R_{1}$ is the Ricci scalar associated with the 2-dimensional metric $h_{A B}$, and now $\frac{1}{2} R_{2}=1$. 
According to our foregoing discussion, any $\mathrm{RC} X$ must be of the form

$$
X=X_{1}+C^{J} \xi_{J}
$$

where $\left\{\xi_{J}, \quad J=1,2,3\right\}$ are the $\mathrm{KV}$ 's that implement the spherical symmetry, $X_{1}=X^{A}(t, r) \partial_{A}$ and $C^{J}$ are constants, $J=1,2$, 3, which can be set equal to zero without loss of generality (since $C^{J} \xi_{J}$ is a $\mathrm{KV}$ of the space-time and therefore a trivial RC). On the other hand, since $\Psi=0$ and $\left(\Phi^{2}\right)_{; A}^{A}=2 \mathbf{e}^{-2 \lambda}\left[1+r\left(\nu^{\prime}-\lambda^{\prime}\right)\right],(27)$ implies

$$
\left\{\mathbf{e}^{-2 \lambda}\left[1+r\left(\nu^{\prime}-\lambda^{\prime}\right)\right]\right\},{ }_{D} X^{D}=0
$$

therefore, the proper RCs of a spherically symmetric space-time whose Ricci tensor is non-degenerate, are of the form

$$
X=X^{t}(t, r) \partial_{t}+X^{r}(t, r) \partial_{r}
$$

and they must satisfy (48) in addition to (12) specialised to the Ricci tensor components given by (43), (44) and (45).

We shall next present two examples: static spherically symmetric spacetimes, and FRW spacetimes.

\subsection{Static spherically symmetric space-times}

Let us consider first the case of static spherically symmetric spacetimes, these are described by (42) where the functions $v$ and $\lambda$ appearing in it depend just on $r, \partial_{t}$ thus being a KV. For the purpose of this paper it is convenient to write the components of the Ricci tensor for this metric as follows [21], [22], 23]

$$
R_{t t} \equiv A(r) \quad R_{r r} \equiv B(r) \quad R_{\theta \theta} \equiv C(r) \quad \text { and } \quad R_{\phi \phi} \equiv \sin ^{2} \theta R_{\theta \theta}
$$

Taking now into account the results of the previous section one has

$$
X=X^{t}(t, r) \partial_{t}+X^{r}(t, r) \partial_{r}
$$

and the (non-trivial) equations arising from (12), are simply:

$$
A^{\prime}(r) X^{r}+2 A(r) X_{, t}^{t}=0
$$




$$
\begin{gathered}
A(r) X_{, r}^{t}+B(r) X_{, t}^{r}=0 \\
B^{\prime}(r) X^{r}+2 B(r) X_{, r}^{r}=0 \\
C^{\prime}(r) X^{r}=0
\end{gathered}
$$

Equation (55) directly implies:

$$
C^{\prime}(r)=0
$$

since otherwise one would have $X^{r}=0$ that would imply, from the remaining equations, $X^{t}=$ constant, thus being a $\mathrm{KV}$ and not a proper $\mathrm{RC}$.

A direct integration of equation (54) gives

$$
X^{r}=\frac{\mathcal{K}(t)}{\sqrt{|B(r)|}}
$$

Now, substituting this result back into eq. (52) and (53), differentiating them with respect to $t$ and $r$, respectively; and equating the crossed derivatives of $X^{t}$, we obtain

$$
\mathcal{K}_{, t t} \frac{\sqrt{|B(r)|}}{A(r)}=\frac{1}{2} \mathcal{K}\left(\frac{A^{\prime}(r)}{A(r) \sqrt{|B(r)|}}\right)^{\prime}
$$

and the following two cases arise:

\subsection{Case I}

$$
\mathcal{K}_{, t t}-\epsilon k^{2} \mathcal{K}=0 ; \quad k=\text { Const }, \quad \epsilon= \pm 1
$$

therefore

$$
\mathcal{K}(t)=\left\{\begin{array}{cc}
a \mathbf{e}^{k t}+b \mathbf{e}^{-k t} & \epsilon=+1 \\
a \sin k t+b \cos k t & \epsilon=-1
\end{array} \mid\right.
$$

and

$$
2 \epsilon k^{2} \frac{\sqrt{|B(r)|}}{A(r)}=\left(\frac{A^{\prime}(r)}{A(r) \sqrt{|B(r)|}}\right)^{\prime}
$$

Substituting these results back into (52), integrating and plugging them back into (53), we find

$$
X^{t}=-\frac{1}{2}\left(\frac{A^{\prime}(r)}{A(r) \sqrt{|B(r)|}}\right) M(t)
$$


where $M(t)=\int \mathcal{K}(t) d t$. and the constant of integration has been set equal to zero without loss of generality.

Thus, for this case a proper $\mathrm{RC}$ is of the form:

$$
X=-\frac{1}{2}\left(\frac{A^{\prime}(r)}{A(r) \sqrt{|B(r)|}}\right)\left(\int \mathcal{K}(t) \mathrm{d} t\right) \partial_{t}+\frac{\mathcal{K}(t)}{\sqrt{|B(r)|}} \partial_{r}
$$

where $\mathcal{K}(t)$ is given by (60), and the components of the Ricci tensor must satisfy (56) and (61).

\subsection{Case II}

$$
\mathcal{K}=\mathcal{S}_{1} t+\mathcal{S}_{2} \quad \mathcal{S}_{1}, \mathcal{S}_{2}=\text { Const }
$$

and

$$
\frac{1}{2} \frac{A^{\prime}(r)}{A(r) \sqrt{|B(r)|}}=\sigma=\text { Const }
$$

Then from (57) and (52-54), one gets after some straightforward calculations:

$$
X=\left\{-\sigma\left(\frac{1}{2} \mathcal{S}_{1} t^{2}+\mathcal{S}_{2} t\right)+\frac{\mathcal{S}_{1}}{2 \sigma} \frac{1}{A(r)}\right\} \partial_{t}+\left(\mathcal{S}_{1} t+\mathcal{S}_{2}\right) \frac{1}{\sqrt{B(r)}} \partial_{r}
$$

As an example of a space-time satisfying the above requirements [24, take for instance

$$
\nu(r)=\frac{1}{2}\left(\frac{r^{4}}{8 r_{0}^{2}}+h \ln \frac{r}{r_{0}}+k\right) \quad \text { and } \quad \lambda(r)=\nu(r)+\ln \frac{r}{r_{0}}
$$

therefore the Ricci components can be written

$$
B(r)=2 \frac{h+1}{r^{2}} \quad C(r)=1 \quad \text { and } \quad A(r)=\text { Const }
$$

where $r_{0}, h$ and $k$ are constants, and we have for the RC:

$$
X^{t}=-c_{4} \sqrt{2(h+1)} \ln r+c_{0} \quad \text { and } \quad X^{r}=\frac{c_{4} t+c_{5}}{\sqrt{2(h+1)}} r
$$


This result invalidates a misleading theorem stated in reference [22], and used in [23]. According to this "theorem", this collineation vector (69) should represent an isometry; however it is easy to see that $X$ does not reduce to a $\mathrm{KV}$ unless $c_{4}=c_{5}=0$.

Since all KV's are naturally RC's and these (if assumed smooth) form a Lie algebra under the usual bracket operation, the Lie bracket of the above RC's with the four KV's the metric admits, must yield in turn RC's; thus

$$
\left[\xi_{I}, \xi\right]=0 \quad \forall I=1,2,3
$$

where $\xi_{I}$ designate the KV's implementing the spherical symmetry, and

$$
\left[\partial_{t}, X\right]=X^{\prime}(\neq 0)
$$

where $X^{\prime}=\frac{c_{4} r}{\sqrt{2(h+1)}}$ is also a proper RC.

A more detailed account of RCs for non-static spherically symmetric space-times will be given in a forthcoming paper.

\subsection{FRW space-times.}

As an example of RC for non-static spherically symmetric metrics, we consider FRW space-times described by [25]:

$$
\mathrm{d} s^{2}=-\mathrm{d} t^{2}+R(t)^{2}\left(\frac{\mathrm{d} r^{2}}{1-k r^{2}}+r^{2} \mathrm{~d} \vartheta^{2}+r^{2} \sin ^{2} \vartheta \mathrm{d} \phi^{2}\right)
$$

Again, using the above notation, we have $\Phi=r R(t)$,

$$
h_{A B} \mathrm{~d} x^{A} \mathrm{~d} x^{B}=-\mathrm{d} t^{2}+R(t)^{2} \frac{\mathrm{d} r^{2}}{1-k r^{2}}
$$

and

$$
h_{\alpha \beta} \mathrm{d} x^{\alpha} \mathrm{d} x^{\beta}=\mathrm{d} \vartheta^{2}+\sin ^{2} \vartheta \mathrm{d} \phi^{2}
$$

Then the Ricci tensor takes the form

$$
\begin{aligned}
& R_{t t}=-3 \frac{\ddot{R}}{R} \\
& R_{r r}=g_{r r} \frac{\Delta}{R^{2}} \\
& R_{\alpha \beta}=g_{\alpha \beta} \frac{\Delta}{R^{2}} \\
& \Delta=2 k+2 \dot{R}^{2}+R \ddot{R}
\end{aligned}
$$


Specializing (12) to the present case, we obtain

$$
\begin{aligned}
& X^{t} R_{r r, t}+X^{r} R_{r r, r}+2 R_{r r} X_{, r}^{r}=0 \\
& X^{t} R_{t t, t}+2 R_{t t} X_{, t}^{t}=0 \\
& R_{t t} X_{, r}^{t}+R_{r r} X_{, t}^{r}=0 \\
& X^{t} R_{\theta \theta, t}+X^{r} R_{\theta \theta, r}=0
\end{aligned}
$$

Thus, we get [11

$$
\begin{aligned}
& X^{t}=c\left(1-k r^{2}\right)^{1 / 2}\left|R_{00}\right|^{-1 / 2} \\
& X^{r}=g(t) r\left(1-k r^{2}\right)^{1 / 2}
\end{aligned}
$$

where $g(t)=-c\left|R_{00}\right|^{-1 / 2}(\dot{\Delta} / 2 \Delta)$, and $c$ is a constant.

\section{ACKNOWLEDGEMENTS}

Two of us (J.C. and U. P.) gratefully acknowledge funding from Postgrado en Astronomía y Astrofísica as well as the warm hospitality of the Laboratorio de Física Teórica, Universidad de Los Andes Mérida, Venezuela, where most of this work was carried out. J.C. acknowledges partial financial support from STRIDE program, Research Project No. STRDB/C/CEN/509/92. The authors wish also to thank the staff of the $S U M A$, the computational facility of the Faculty of Science (Universidad de Los Andes).

\section{References}

[1] G.H. Katzin, J. Levine and W.R. Davis, J. Math. Phys. 10, 617 (1969).

[2] Hojman, S., Núñez, L., Patiño, A., and Rago, H. J. Math. Phys 27, 281 (1986).

[3] Bedran, M.L.,and Lesche, B. J. Math. Phys 27, 2360 (1986).

[4] Aichelburg, P. (1970) J. Math. Phys. 11, 2458.

[5] L.H. Green, L.K. Norris, D.R. Oliver and W.R, Davis, Gen. Rel. Grav. 8, 731 (1977). 
[6] Coley, A.A., and Tupper, B.O.J. (1989) J. Math. Phys. 30, 2616.

[7] D.R. Oliver and W.R, Davis, Gen. Rel. Grav. 8, 905 (1977).

[8] M. Tsamparlis and D.P. Mason, J. Math. Phys. 31, 1707 (1990).

[9] K.L. Duggal, J. Math. Phys. 33, 2989 (1992).

[10] L. Aulestia, L. Núñez, A. Patiño, H. Rago and L. Herrera, Nuov. Cim. B 80, 133 (1984).

[11] L. Núñez, U. Percoco and V. M. Villalba, J. Math. Phys. 31, 137 (1990).

[12] A. Melfo, L. A. Núñez, U. Percoco and V. Villalba, J. Math. Phys. 33, 2558 (1992).

[13] A. Melfo and L.A. Núñez, Gen. Rel. Grav. 24, 1125 (1992).

[14] R. Bertolotti, L. A. Núñez and U. Percoco "Computer Algebra and Collineation Vectors in General Relativity" Preprint Laboratorio de Física Teórica, Universidad de los Andes (1995).

[15] J. Carot, J. da Costa and E. G. L. R. Vaz, J. Math. Phys. 35, 4832 (1994).

[16] G.S. Hall, J. Math. Phys., 31, 1198, (1990)

[17] G.S. Hall, I. Roy and E. G. L. R. Vaz, "Ricci and Matter Collineations in Spacetimes" Preprint University of Aberdeen (1995).

[18] J. Carot and J. da Costa Class. Quantum Grav. 10, 461 (1993).

[19] B. Haddow and J. Carot Class. Quantum Grav., 13 (1996) 289

[20] D. Kramer, H. Stephani, E. Hearlt, and M. A. H. MacCallum, Exact Solutions of Einstein Field Equations (Cambridge University, Cambridge, 1980).

[21] A. H. Bokhari, and A. Qadir, J. Math. Phys. 34, 3543 (1993).

[22] M. Jamil Amir, A. H. Bokhari, and A. Qadir, J. Math. Phys. 35, 3005 (1994). 
[23] T. B. Farid, A. Qadir and M. Ziad J. Math. Phys. 36, 5812 (1995)

[24] R. Bertolotti, G. Contreras, L. A. Núñez, U. Percoco and J. Carot J. Math. Phys., 37,1086-1088 (1996).

[25] S. Weinberg, Gravitation and Cosmology (Wiley, New York 1972). 